\title{
BMJ Open Optimal target blood pressure in critically ill adult patients with vasodilatory shock: a protocol for a systematic review and meta-analysis
}

\author{
Satoshi Fukui, ${ }^{1}$ Koki Higashio, ${ }^{1}$ Shuhei Murao, ${ }^{2}$ Akira Endo, ${ }^{3}$ Takasu Akira, ${ }^{4}$ \\ Kazuma Yamakawa
}

To cite: Fukui S, Higashio K, Murao S, et al. Optimal target blood pressure in critically ill adult patients with vasodilatory shock: a protocol for a systematic review and meta-analysis. BMJ Open 2021;11:e048512. doi:10.1136/ bmjopen-2020-048512

- Prepublication history for this paper is available online. To view these files, please visit the journal online (http://dx.doi. org/10.1136/bmjopen-2020048512).

Received 29 December 2020 Revised 04 March 2021 Accepted 08 March 2021

Check for updates

(C) Author(s) (or their employer(s)) 2021. Re-use permitted under CC BY-NC. No commercial re-use. See rights and permissions. Published by BMJ.

${ }^{1}$ Faculty of Medicine, Osaka Medical College, Takatsuki,

Osaka, Japan

${ }^{2}$ Department of Traumatology and Acute Critical Medicine, Osaka University School of Medicine Graduate School of Medicine, Suita, Osaka, Japan

${ }^{3}$ Trauma and Acute Critical Care Center, Tokyo Medical and Dental University, Bunkyo-ku, Tokyo, Japan

${ }^{4}$ Department of Emergency

Medicne, Osaka Medical

College, Takatsuki, Osaka, Japan

Correspondence to

Dr Kazuma Yamakawa;

k.yamakawa0911@gmail.com

\section{ABSTRACT}

Introduction The optimal target of mean arterial pressure (MAP) for better outcomes in patients with vasodilatory shock remains a matter of debate. Although catecholamines are generally used to maintain target blood pressure in hypotensive patients with vasodilatory shock, the adverse effects of catecholamines must also be considered. We will perform a systematic review and meta-analysis of randomised controlled trials (RCTs) to assess the certainty of evidence determining the optimal target of MAP control for patients with vasodilatory shock in critically ill settings.

Methods and analysis This study protocol was registered in the University Hospital Medical Information Network Clinical Trials Registry. We will include only RCTs that evaluated the two different comparators for target MAP to be maintained for clinical outcomes of allcause mortality: organ dysfunction and adverse events in critically ill adult patients with vasodilatory shock. We will search the electronic bibliographic databases of MEDLINE, EMBASE and the Cochrane Central Register of Controlled Trials in November 2020. Two reviewers will independently screen titles and abstracts, perform full article reviews and extract study data. We will report study characteristics and assess methodological quality using the Cochrane Riskof-Bias 2 tool. If pooling is appropriate, we will calculate relative risks with $95 \%$ Cls for all outcome measures. Clinical and methodological subgroup and sensitivity analyses will be performed to explore heterogeneity. Overall certainty of evidence will be evaluated using the Grading of Recommendations Assessment, Development and Evaluation approach.

Ethics and dissemination This study will not involve primary data collection, and formal ethics approval will therefore not be required. We aim to publish this systematic review in a peer-reviewed journal. Trial registration number UMIN000042624.

\section{INTRODUCTION}

Optimisation of systemic oxygen metabolism and regional tissue perfusion of the vital organs is a key component of treatment in critically ill settings. Among the haemodynamic parameters, mean arterial pressure (MAP) is a common indicator of perfusion

\section{Strengths and limitations of this study}

- This systematic review will be the latest report to answer the clinical question of what the optimal target should be for mean arterial pressure (MAP) control in patients with vasodilatory shock in critically ill settings.

- The supposed major limitations in this review will be the clinical heterogeneity of the included trials, diversity of the targeted population with vasodilatory shock and definition of the intervention and control arms.

- This systematic review will be carried out using appropriate methodologies and quality assessment tools according to the Grading of Recommendations Assessment, Development and Evaluation approach.

- The results will be directly useful in helping clinicians to better determine the target MAP for goaldirected management of critically ill patients with vasodilatory shock.

to the vital organs and is frequently used in the clinical setting. Although the international sepsis guidelines recommended an initial target for MAP of $65 \mathrm{~mm} \mathrm{Hg}$ in septic shock patients, ${ }^{1}$ we acknowledge that there is no clear evidence for the use of this value. The optimal target of MAP for better outcomes in patients with vasodilatory shock remains a matter of debate. ${ }^{2-4}$ Although catecholamines are generally used to maintain the target blood pressure in hypotensive patients with vasodilatory shock, the adverse effects of catecholamines must also be considered. While maintaining the usual level of blood pressure might play a crucial role in improving the perfusion of vital organs and thus avoiding shockassociated organ dysfunction, ${ }^{5}$ especially in patients with chronic hypertension or elderly patients, ${ }^{6}$ cardiologic adverse effects related to catecholamines such as arrhythmias and tachycardia are a concern. The net 
effects or balances between such benefit and harm of catecholamines can affect patient outcome in the clinical setting.

Previously, several studies were published regarding the optimal target of MAP in critically ill settings. ${ }^{7}$ Generally, management with an initial target MAP of $65 \mathrm{~mm} \mathrm{Hg}$ or higher has been considered as good practice in critically ill patients. ${ }^{89}$ However, two major randomised controlled trials (RCTs), the SEPSISPAM ${ }^{10}$ and OVATION trials, ${ }^{11}$ which evaluated optimal MAP targets, showed that maintaining a higher MAP by administration of vasopressors may increase the risk of death in patients older than 65 years. These RCTs and subsequent meta-analyses ${ }^{12-14}$ suggested that management targeting a higher MAP might not always be beneficial in chronically hypertensive patients.

As mentioned earlier, the optimal target of blood pressure control in vasodilatory shock or sepsis remains unclear due to limited evidence. Especially, it is still unknown whether maintaining a higher MAP may effect a decrease in organ damage due to sufficient oxygen delivery or an increase in adverse events due to vasopressor use. Recently, the novel large-scale RCT by Lamontagne et al called the '65 Trial' was published in $J A M A,{ }^{15}$ but it has not yet been included in any systematic reviews or meta-analyses. Here, we describe our planned updated systematic review and meta-analysis of RCTs to assess the certainty of evidence determining the optimal target of MAP control for patients with vasodilatory shock in critically ill settings.

\section{METHODS AND ANALYSIS}

\section{Protocol registration}

This study protocol was registered in the University Hospital Medical Information Network (UMIN) Clinical Trials Registry (https://www.umin.ac.jp/ctr/index-j. htm) (Registration No. UMIN000042624). The protocol follows the Preferred Reporting Items for Systematic Reviews and Meta-Analyses Protocols (PRISMA-P) statements, ${ }^{16}$ and the systematic review and meta-analysis will be reported according to the PRISMA statement. ${ }^{17}$

\section{Focused review questions}

The objective of this review was to determine the best clinical practice for haemodynamic stabilisation in terms of the optimal target blood pressure in critically ill adult patients with vasodilatory shock.

\section{Types of studies}

We will include only RCTs that studied the optimal target blood pressure in critically ill adult patients with vasodilatory shock. Articles including cohort studies, case-control studies, experimental animals, narrative reviews, correspondence, case reports, expert opinions and editorials will be excluded.

\section{Condition or domain being studied}

The study domain will include adult patients with vasodilatory shock in critically ill settings.

\section{Types of participants}

We will include adult patients with vasodilatory shock caused by any underlying disease such as sepsis, severe acute pancreatitis, the postoperative state and other causes. We will include studies evaluating critically ill adult patients aged 16 years or older. Studies done with animals will be excluded from this review.

\section{Types of interventions and comparators}

We will include studies that describe the two different comparators for target MAP to be maintained: high-target MAP group and low-target MAP group. The definitions of actual target arterial pressure in both groups will not matter and may vary across studies.

\section{Types of outcome assessments}

The primary outcome measure will be all-cause mortality. In case multiple mortality outcomes are reported, the mortality outcome closest to 28-day outcome will be selected. We will also assess the outcome measures of intensive care unit mortality or in-hospital mortality. As secondary outcome measures, the number of patients with organ dysfunction (respiratory and renal) and serious adverse events related to the study interventions will be assessed.

\section{Search strategy}

We will search the following databases for relevant studies: MEDLINE (via PubMed), EMBASE and the Cochrane Central Register of Controlled Trials. We have developed a search strategy using a combination of keywords and Medical Subject Headings (MeSH)/EMTREE terms. The search strategy in MEDLINE is as follows: ('shock' (MeSH Terms) OR 'vasopressor*' (Title/Abstract)) AND ('Blood Pressure' (MeSH Terms) OR 'Blood Pressure' (Title/ Abstract)) AND (('randomized controlled trial' (Publication Type) OR 'controlled clinical trial' (Publication Type) OR 'randomized' (Title/Abstract) OR 'placebo' (Title/Abstract) OR 'clinical trials as topic' (MeSH Terms:noexp) OR 'randomly' (Title/Abstract) OR 'trial' (Title)) NOT ('animals' (MeSH Terms) NOT 'humans' (MeSH Terms))). We will screen the reference lists of all relevant papers for additional studies. We will also contact relevant authors for further details and information on ongoing or unpublished trials. Our MEDLINE search strategy will be adapted appropriately for searches in the other two databases. No language or time restrictions on electronic searches will be applied. The planned date of our systematic search is November 2020.

\section{Citation management and screening}

Citations will be stored, and duplicates will be removed using EndNote software (Thomson Reuters, Toronto, Ontario, Canada). Studies will be screened initially according to title and abstract by two authors (SF and $\mathrm{KH}$ ) 
independently, and those not meeting the criteria will be discarded. Disagreements will be resolved by discussion and referral to a third author (KY) if necessary. After this initial stage, the full text of all remaining studies will be reviewed, and disagreements will be resolved in the same way as in the initial screening. We will use the Rayyan QCRI website (http://rayyan.qcri.org) ${ }^{18}$ in this screening process. We will document the study selection process in a PRISMA flow diagram.

\section{Data extraction}

Two authors (SF and $\mathrm{KH}$ ) will extract the study characteristics from each included study, including data on the assessment of quality and the investigation of heterogeneity, and transfer that information into a study-specific format. Adjudication by a third author (KY) will be used if necessary. Efforts will be made to contact the authors of the primary studies to provide missing data where necessary.

\section{Assessment of risk of bias}

Independent reviewers ( $\mathrm{SF}$ and $\mathrm{KH}$ ) will assess the risk of bias in individual trials as the methodological quality of the articles. Disagreements will be resolved by discussion and consensus and the participation of a third author (KY) when necessary. To evaluate the risk of bias in the individual RCTs, we will use the revised uniform criteria of the Cochrane risk-of-bias tool for randomised trials version 2 (RoB 2).$^{19}$ For each domain, we will assign a judgement regarding the risk of bias as high, low or some concerns. We will attempt to contact the trial corresponding author for clarification when insufficient detail is reported to assess the risk of bias.

\section{Data synthesis}

Statistical analyses will be performed using Review Manager V.5.4 software (Nordic Cochrane Centre, Cochrane Collaboration) and STATA software V.14.0 (STATA Corporation). For each included trial, we will calculate the relative risks with $95 \%$ CIs for all outcome measures. We will perform both fixed-effects analysis using the Mantel-Haenszel method and random-effects analyses using the DerSimonian and Laird estimator, reporting the most conservative summary estimate with the broadest CI.

\section{Assessment of heterogeneity}

We will investigate heterogeneity initially by visual examination of forest plots. Statistical heterogeneity will be evaluated informally from forest plots of the study estimates and more formally using the $\chi^{2}$ test ( $\mathrm{p}$ value $<0.1=$ significant heterogeneity) and $I^{2}$ statistic $\left(I^{2}>50 \%=\right.$ significant heterogeneity).

If sufficient studies are available, we will undertake sensitivity and subgroup analyses to explore the sources of potential heterogeneity across trials. The analysis will be performed using the following as covariates: age, sex, severity of disease, sepsis or not, existence of chronic hypertension and targeted blood pressure.

\section{Assessment of publication biases}

If a sufficient number of studies are identified, we will create funnel plots for mortality in which the log RRs are plotted against their SEs, and we will test the symmetry of the funnel plots using Begg's rank correlation test and Egger's linear regression test. We will interpret this analysis with caution given the lack of statistical power of these tests.

\section{Rating of the certainty of evidence using the Grading of Recommendations Assessment, Development and Evaluation (GRADE) approach}

The risk of systematic errors (bias) and random errors will be assessed, and the overall certainty of evidence will be evaluated using the GRADE approach.

\section{Patient and public involvement}

No patient will be involved in the design or planning of the study.

\section{DISCUSSION}

We manage critically ill patients with vasodilatory shock using fluid challenges and vasoconstriction agents and usually perform a goal-directing strategy by setting a target blood pressure. However, a fundamental consensus regarding the target blood pressure has not been achieved. As we work on this clinical question, it remains a challenge for clinicians to balance the risk of hypotension with the side effects of vasopressors. The previously published SEPSISPAM ${ }^{10}$ and OVATION ${ }^{11}$ trials evaluated the association between MAP targets and 28-day mortality in patients older than 18 and 16 years, respectively, and could not show a survival benefit for the strategy targeting high blood pressure over $65 \mathrm{mmHg}$. The pooled analysis $^{12}$ evaluating the data from these two works suggested that the mortality risk related to the high blood pressure target strategy rose according to the increase in patient age. Other mortality studies have also been done, ${ }^{6}$ but whether high or low MAP should be the target is still inconclusive. Conducting meta-analyses and systematic reviews of the evidence related to this clinical question may lead to further research in critical care fields and may allow clinicians to set specific blood pressure targets for elderly patients with vasodilatory shock.

We acknowledge potential limitations. First, we will include all patients with vasodilatory shock. Although this choice of patient selection will contribute to increasing the pooled sample size, it might introduce clinical heterogeneity. However, we will conduct a detailed preplanned subgroup analysis according to underlying diseases to evaluate heterogeneity between different types of shock. Second, the supposed definitions of the intervention and control arms could be different between groups (ie, the targeted MAP of higher/lower groups). As with the first limitation, we will consider this heterogeneity cautiously. Otherwise, we believe that the results will be directly useful in helping clinicians to make better decisions when 
targeting MAP for goal-directed management in critically ill patients with vasodilatory shock.

\section{ETHICS AND DISSEMINATION}

Approval from an ethics committee will not be required as this systematic review will use publicly available data without directly involving human participants. Our findings will be presented at relevant scientific conferences and disseminated through publication in a peer-reviewed journal.

Contributors $\mathrm{KY}$ is a guarantor and contributed to the conception of the study. The manuscript protocol was drafted by SF and KY and was revised by SM, AE and TA. The search strategy was developed by KY and SM and will be performed by SF and $\mathrm{KH}$, who will also independently screen the potential studies. SF and KH will independently extract data from the included studies, assess the risk of bias and complete the data synthesis. KY will arbitrate in cases of disagreement and ensure the absence of errors. All authors approved the publication of this protocol.

Funding The authors have not declared a specific grant for this research from any funding agency in the public, commercial or not-for-profit sectors.

Competing interests None declared.

Patient and public involvement Patients and/or the public were not involved in the design, or conduct, or reporting or dissemination plans of this research.

Patient consent for publication Not required.

Provenance and peer review Not commissioned; externally peer reviewed.

Open access This is an open access article distributed in accordance with the Creative Commons Attribution Non Commercial (CC BY-NC 4.0) license, which permits others to distribute, remix, adapt, build upon this work non-commercially, and license their derivative works on different terms, provided the original work is properly cited, appropriate credit is given, any changes made indicated, and the use is non-commercial. See: http://creativecommons.org/licenses/by-nc/4.0/.

ORCID iD

Kazuma Yamakawa http://orcid.org/0000-0003-2999-4021

\section{REFERENCES}

1 Rhodes A, Evans LE, Alhazzani W, et al. Surviving sepsis campaign: international guidelines for management of sepsis and septic shock: 2016. Intensive Care Med 2017;43:304-77.

2 Russell JA. Personalized blood pressure targets in shock: what If your normal blood pressure is "low"? Am J Respir Crit Care Med 2020;202:10-12.
3 Leone M, Asfar P, Radermacher P, et al. Optimizing mean arterial pressure in septic shock: a critical reappraisal of the literature. Crit Care 2015;19:101-7.

4 Corrêa TD, Jakob SM, Takala J. Arterial blood pressure targets in septic shock: is it time to move to an individualized approach? Crit Care 2015;19:4-5.

5 Fiorese Coimbra KT, de Freitas FGR, Bafi AT, et al. Effect of increasing blood pressure with noradrenaline on the microcirculation of patients with septic shock and previous arterial hypertension. Crit Care Med 2019;47:1033-40.

$6 \mathrm{Xu} \mathrm{J-Y,} \mathrm{Ma} \mathrm{S-Q,} \mathrm{Pan} \mathrm{C,} \mathrm{et} \mathrm{al.} \mathrm{A} \mathrm{high} \mathrm{mean} \mathrm{arterial} \mathrm{pressure} \mathrm{target} \mathrm{is}$ associated with improved microcirculation in septic shock patients with previous hypertension: a prospective open label study. Crit Care 2015;19:130.

7 Bourgoin A, Leone M, Delmas A, et al. Increasing mean arterial pressure in patients with septic shock: effects on oxygen variables and renal function. Crit Care Med 2005;33:780-6.

8 Maheshwari K, Nathanson BH, Munson SH, et al. The relationship between ICU hypotension and in-hospital mortality and morbidity in septic patients. Intensive Care Med 2018;44:857-67.

9 Varpula M, Tallgren M, Saukkonen K, et al. Hemodynamic variables related to outcome in septic shock. Intensive Care Med 2005;31:1066-71.

10 Asfar P, Meziani F, Hamel J-F, et al. High versus low bloodpressure target in patients with septic shock. N Engl J Med 2014;370:1583-93.

11 Lamontagne F, Meade MO, Hébert PC, et al. Higher versus lower blood pressure targets for vasopressor therapy in shock: a multicentre pilot randomized controlled trial. Intensive Care Med 2016;42:542-50.

12 Lamontagne F, Day AG, Meade MO, et al. Pooled analysis of higher versus lower blood pressure targets for vasopressor therapy septic and vasodilatory shock. Intensive Care Med 2018;44:12-21.

13 Hylands M, Moller MH, Asfar P. Une revue systématique des cibles de tension artérielle sous vasopresseurs chez des adultes gravement malades atteints d'hypotension. Can J Anesth 2017;64:703-15.

14 D'Aragon F, Belley-Cote EP, Meade MO, et al. Blood pressure targets for vasopressor therapy: a systematic review. Shock 2015;43:530-9.

15 Lamontagne F, Richards-Belle A, Thomas K, et al. Effect of reduced exposure to vasopressors on 90-day mortality in older critically ill patients with vasodilatory hypotension: a randomized clinical trial. JAMA 2020;323:938-49.

16 Shamseer L, Moher D, Clarke M, et al. Preferred reporting items for systematic review and meta-analysis protocols (PRISMA-P) 2015: elaboration and explanation. BMJ 2015;350:g7647.

17 Liberati A, Altman DG, Tetzlaff J, et al. The PRISMA statement for reporting systematic reviews and meta-analyses of studies that evaluate healthcare interventions: explanation and elaboration. $B M J$ 2009;339:b2700.

18 Ouzzani M, Hammady H, Fedorowicz Z, et al. Rayyan-a web and mobile APP for systematic reviews. Syst Rev 2016;5:210.

19 Sterne JAC, Savović J, Page MJ, et al. Rob 2: a revised tool for assessing risk of bias in randomised trials. BMJ 2019;366:14898. 